\title{
Plasma visfatin levels and insulin sensitivity or resistance relationship in type 2 diabetes
}

\author{
Shatha Abdul Wadood AL- Shammaree
}

Department of Chemistry, College of Science, University of Baghdad, Baghdad, Iraq

*Correspondence to: Shatha Abdul Wadood AL-Shammaree (e-mail: shath_a@yahoo.com).

(Submitted: 29 August 2017 - Revised version received: 07 September 2017 - Accepted: 16 October 2017 - Published online: 26 December 2017)

\begin{abstract}
Objective Many studies reported inconsistent results about the connection of visfatin with diabetes and insulin resistance (IR), and also the role of visfatin in the pathogenesis of diabetes are still under debate. This study aimed to evaluate the visfatin levels in patients with type 2 diabetes (T2DM) compared to non-diabetic age-matched subjects and explore the relationship between visfatin and insulin sensitivity or resistance. Methods A total of 85 subjects were enrolled in this study; 60 patients with T2DM who were sub-divided into two groups according to levels of $I R$ ( $I R<2.5$ and $>2.5$ groups). Also, 25 non-diabetic healthy subjects matched for age, body mass index, and lipid profile were included as a control group. Physical and clinical examinations were done for each participant. Visfatin levels were measured using enzyme-linked immune sorbent assay. IR and quantitative insulin sensitivity check index (QUICKI) were calculated from fasting plasma glucose and insulin.

Results Significant higher visfatin level and IR were found in diabetic patients in comparison to control group $(P<0.05)$. No significant difference was found in visfatin level between diabetic patients with $\mathbb{R}<2.5$ and control group, while, visfatin level in diabetic patients with $\mathbb{R}>2.5$ was significantly higher in comparison to that of the group with $\mathbb{R}<2.5$ and control group. Significant negative correlation was found between visfatin levels and QUICKI in the diabetic patients' group with $\mathbb{R}<2.5(r=-0.324, P<0.05)$. Highly significant negative correlation was found between QUICKI and IR in control and diabetic patients with $I R<2.5$, while this correlation disappears in IR $>2.5$ group.

Conclusion An inverse relationship between visfatin levels and insulin sensitivity was found. Also, the correlation between insulin sensitivity and resistance differs depending on their levels. This study suggests that visfatin may play a significant regulatory role at elevated glucose levels, spatially when IR is more than 2.5 .

Keywords visfatin, type 2 diabetes, insulin resistance, insulin sensitivity
\end{abstract}

\section{Introduction}

Visfatin is an adipokine secreted from the adipose tissue that was formerly described Nicotinamide phosphoribosyl transferase (Nampt). ${ }^{1}$ It is also secreted from lymphocytes, leukocytes, muscles, and hepatocytes. ${ }^{2,3}$ It was found that visfatin is expressed as a response to inflammation from macrophages of adipose tissue rather than from adipocytes. ${ }^{4,5}$ Although adipose tissue is considered an endocrine tissue, it is now believed that visfatin actions can be endocrine, paracrine, and autocrine as well. The autocrine effects of visfatin in the liver may play an important role in regulating insulin sensitivity. ${ }^{6}$ Visfatin is involved in regulation of the cell cycle. ${ }^{7}$ It is secreted by activated lymphocytes and is upregulated in neutrophils and monocytes after exposure to inflammatory stimuli. It also promotes the growth of $\mathrm{B}$-cell precursors and is therefore known as a pre-B-cell colony-enhancing factor. ${ }^{8}$

Although, visfatin physiological role in human beings still largely unrevealed; many studies showed that visfatin has many functions such as a cytokine, ${ }^{19}$ a hormone ${ }^{1}$ and lately, as an enzyme (the rate-limiting enzyme Nampt in the NAD biosynthesis). ${ }^{10}$ Various medical conditions were correlated with visfatin levels through its anti-diabetic, ${ }^{1}$ anti-tumor, ${ }^{11}$ anti-hyperlipidemia, ${ }^{12}$ and pro-inflammatory properties. ${ }^{9}$ This is due to its role in the modulation of insulin sensitivity, insulin resistance (IR) in diabetes, dyslipidemia, inflammation and atherosclerosis. ${ }^{13}$

In diabetic patients, irregular feedback mechanism in the hypothalamic-pituitary axis was found through the decrease in $\mathrm{LH}$ and FSH levels due to high glucose levels and IR. ${ }^{14}$

Hammarstedt et al. (2006) ${ }^{15}$ reported a double increase in plasma visfatin levels in T2DM compared with controls, which is similar to other studies. ${ }^{12,16,17}$ However, others found no correlation between circulating visfatin, insulin, and glucose. ${ }^{18,19}$ Lopez-Bermejo et al. $(2006)^{20}$ reported a significant increase in levels of visfatin in patients with T1DM compared with T2DM or non-diabetic subjects. The correlation of visfatin levels with obesity and IR was revealed by Rabo et al. $(2013)^{16}$ study who found that they have higher significant positive correlation in both obese and non-obese diabetic patients with a significant positive correlation with IR. This inconsistency in the results of many researches regarding the correlation of visfatin with diabetes and IR leads to more investigation. This study aimed to determine the plasma visfatin level in patients with T2DM and in control, also, explore the relationship between plasma visfatin and insulin sensitivity/resistance.

\section{Materials and Methods}

The case control study enrolled 16 patients with T2DM who attended the National Diabetes Center for diagnosis, treatment, and follow-up at Al-Mustansiyria University in Baghdad, Iraq. The diagnosis was based on the criteria of the World Health Organization (WHO) (FBG $\geq 126 \mathrm{mg} / \mathrm{dl}$ ). ${ }^{21}$ The duration of the disease is between 4 and 8 years, and all patients were on oral glucose lowering treatment. The mean level of age and BMI were ( $40 \pm 6.23$ years; $29.15 \pm 3.21 \mathrm{~kg} / \mathrm{m}^{2}$, respectively). Twenty five healthy subjects were included as a control group who matched to patients group in age, and BMI $\left(37.90 \pm 8.60\right.$ years; $27.41 \pm 4.01 \mathrm{~kg} / \mathrm{m}^{2}$, respectively). The patients were selected with normal lipid profile to exclude the effect of lipogenesity on visfatin level. A written approval was taken from ethical and scientific committee and from each participant. Any patient with any complication of diabetes or any other disease that has any effect on the studied parameters was excluded. 


\section{Samples}

Five $\mathrm{ml}$ of fasting venous blood was withdrawn from each participant after physical and clinical examination. Renal function was determined (uric acid and creatinine) using an autoanalyzer device (Biolabo, France). Fasting plasma glucose was determined using the oxidase method. Fasting insulin (FI) was measured using a competitive enzyme immunoassay kit (Randox; UK). IR was calculated according to the formula:

IR $=$ Fasting plasma insulin $(\mathrm{mIU}) \times$ fasting plasma glucose $(\mathrm{mM} / \mathrm{l})] / 22.5$.

The patient group was sub-divided according to IR levels to $<2.5$ and $>2.5$ groups. ${ }^{22}$ The determination of plasma visfatin level was done using RayBio ${ }^{\star}$ Visfatin Enzyme Linked Immunosorbent assay (EIA) Kit. The sensitivity of the visfatin assay is $0.778 \mathrm{ng} / \mathrm{ml}$, with Intra-Assay: $\mathrm{CV}<10 \%$, and InterAssay: CV $<15 \%$. Quantitative insulin sensitivity check index (QUICKI) was used to measure insulin sensitivity from fasting blood glucose and insulin using the following formula: ${ }^{22}$

$$
\text { QUICKI }=1 /\left[\log \left(I_{o}\right)+\log \left(G_{0}\right)\right]
$$

where $I_{o}$ is the fasting plasma insulin, and $G_{o}$ is the fasting plasma glucose.

The documented QUICKI level is: $(0.382 \pm 0.007)$ for non-obese, $(0.331 \pm 0.010)$ for obese and $(0.304 \pm 0.007)$ for diabetic individuals. ${ }^{23}$

\section{Statistical Analysis}

Statistical analysis was performed using a standard statistical package (SPSS) for Windows, version 21; Chicago, IL, USA). The data were expressed as mean \pm SD. Two-way student's $t$-test was used for comparison between two groups. The post has a one-way analysis of variance (ANOVA) was used for comparison between groups. The association of visfatin with QUICKI was examined by Pearson correlation analysis, as well as the correlation between IR and QUICKI. When $P$ value less than 0.05 ; it is statistically significant.

\section{Results}

The characteristics and some clinical measurements are presented in Table 1. Body mass index, FI, uric acid, and creatinine showed no significant differences between patients and control groups $(P>0.05)$ while significant increase was found in patient group when compared to control group for FPG, and IR, $(P<0.05)$.

The plasma visfatin level was significantly higher in the diabetic patient group compared with control group (9.52 \pm 1.56 vs. $8.28 \pm 0.62 \mathrm{ng} / \mathrm{ml}, P=0.0001$, respectively).

Patients were sub-grouped, depending on IR levels, into IR $<2.5$ and IR $>2.5$ groups. The QUICKI level in both groups showed a significant decrease when compared to control group $(0.28 \pm 0.015$ and $0.31 \pm 0.015$ vs. $0.34 \pm 0.013, P=0.042$ respectively) as presented in Table 2.

Visfatin level in a group of patients with IR $>2.5$ show significant higher levels in comparison to that of patient group with IR $<2.5$ and control group $(9.17 \pm 1.10$ vs. $9.12 \pm 1.02$ and $8.28 \pm 0.62, P=0.017$, respectively), while no significant difference was found between patient group with $\mathrm{IR}<2.5$ and control group $(P>0.05)$ as presented in Table 2 and Fig. 1.

Pearson correlation between visfatin and FPG, FI, IR, creatinine, and uric acid showed no significant differences, while a significant negative correlation was found with QUICKI ( $r=-0.324, P<0.05)$, as presented in Fig. 2 .

A highly negative significant correlation was found between QUICKI and IR for the control group $(r=0.952, P<$ $0.01)$ while, the weak negative significant correlation was observed in the patient group with IR $<2.5(r=0.473, P<$ $0.05)$ but this correlation disappear in the patient group with IR $>2.5$ as shown in Fig. 3.

\section{Discussion}

Plasma visfatin levels were higher in diabetic patients compared to control group which is consistent with other studies ${ }^{15,20,24}$ and counteract with other's results ${ }^{25-27}$ who found lower visfatin levels in T2DM when compared with matched non-diabetic subjects.

The increase in circulating visfatin levels by hyperglycemia is not clear until now, but it may be due to oxidative stress, increased apoptosis, or destruction of B lymphocytes. It was suggested that the elevation in levels of circulating visfatin is a compensatory mechanism for the decreased insulin sensitivity in patients with T2DM. This is in agreement with the results of other research. ${ }^{16,28}$

However, Yaturu et al. (2012) measured visfatin levels in T2DM patients who were under pioglitazone treatment. They elucidate the decrease in visfatin levels, which may be due to the treatment. ${ }^{27}$ Their result confirms what McGee

\section{Table 1. Mean ( $\mathbf{t} \mathbf{S D})$ of some characteristic and clinical measurements in patients and control groups}

\begin{tabular}{lccc}
\hline Parameters & Control group & Patients group & P value \\
\hline No. & 25 & 60 & - \\
Age (year) & $37.90 \pm 8.60$ & $40.25 \pm 6.23$ & $>0.05$ \\
Sex (Male/Female) & $11 / 14$ & $35 / 25$ & - \\
Duration (year) & - & $6.4 \pm 2.11$ & - \\
Uric acid (mmol/L) & $341.5 \pm 110.8$ & $374.9 \pm 104.1$ & 0.196 \\
Creatinine (mg/dl) & $0.99 \pm 0.03$ & $1.19 \pm 0.06$ & 0.43 \\
FBG (mg/dl) & $89.54 \pm 10.93$ & $219.91 \pm 94.32$ & 0.014 \\
Fl ( $\mu l U / m l)$ & $10.33 \pm 2.45$ & $20.96 \pm 7.92$ & 0.067 \\
IR & $1.43 \pm 0.73$ & $5.34 \pm 2.03$ & 0.01 \\
Visfatin (ng/ml) & $8.28 \pm 0.62$ & $9.52 \pm 1.56$ & 0.0001 \\
\hline
\end{tabular}

Significant difference is when $P$ value less than 0.05 using independent student's $t$-test. BMI, Body mass index; FPG, fasting plasma glucose; $\mathrm{Fl}$, fasting insulin; IR, insulin resistance.

Table 2. QUICKI and visfatin levels (expressed as mean \pm SD) in patients sub-grouped according to IR levels in comparison to control group

\begin{tabular}{lcccc}
\hline Parameters & $\begin{array}{c}\text { Control } \\
\text { group }\end{array}$ & $\begin{array}{c}\text { IR }>\mathbf{2 . 5} \\
\text { group }\end{array}$ & $\begin{array}{c}\text { IR }<\mathbf{2 . 5} \\
\text { group }\end{array}$ & P value \\
\hline No. & 25 & 30 & 30 & - \\
QUICKI & $0.34 \pm 0.013$ & $0.28 \pm 0.015$ & $0.31 \pm 0.015$ & 0.042 \\
Visfatin ng/ml & $8.28 \pm 0.62$ & $9.03 \pm 1.02$ & $7.96 \pm 1.10$ & 0.017 \\
\hline
\end{tabular}

The $P$ value less than 0.05 is considered significant using post hoc ANOVA analysis. IR, insulin resistance; QUICKI, Quantitative insulin sensitivity check index. 


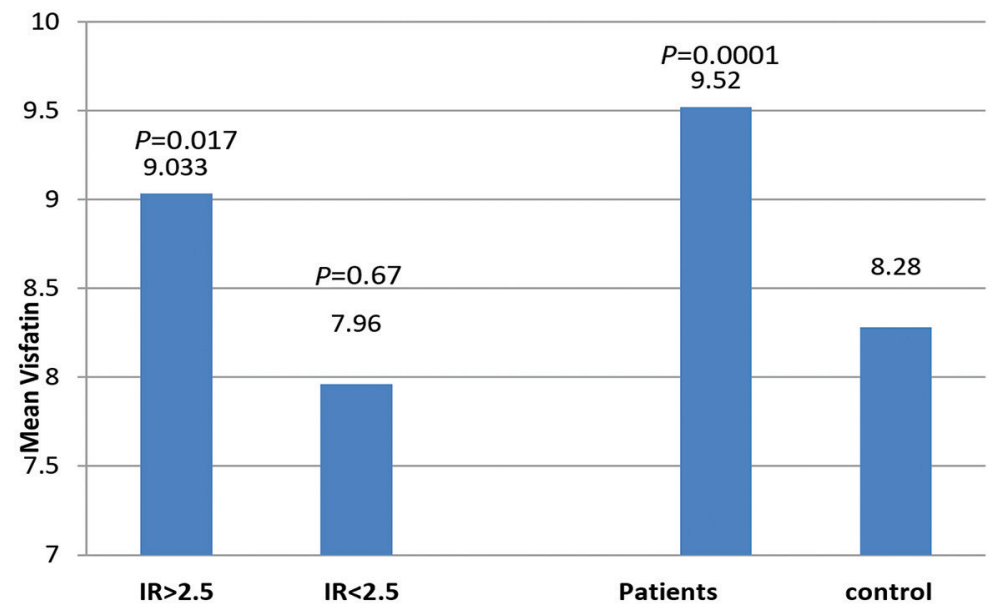

Fig 1. Mean level and significant differences of visfatin in patients groups in comparison to control group.

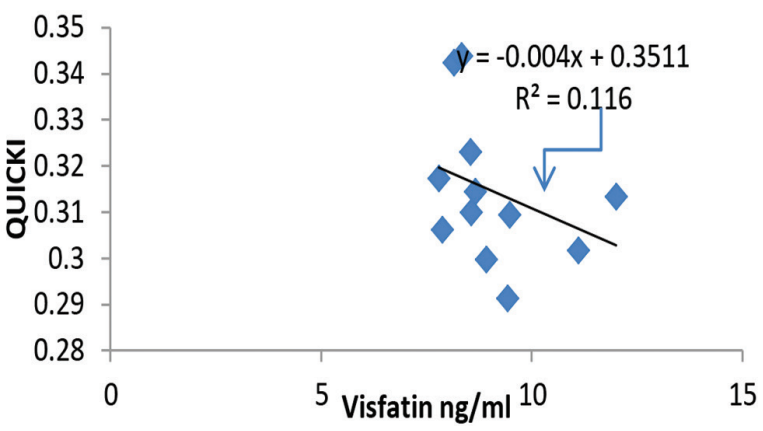

Fig 2. The Pearson correlation between visfatin and QUICKI in diabetic patients with $I R<2.5$.

et al., (2011) had previously found when they used rosiglitazone for T2DM treatment. ${ }^{29}$

The plasma visfatin increase as fasting blood glucose and IR increase, but it didn't reach significance level. Previous studies documented the role of this hormone as an insulin-resembling effect in lowering blood glucose through binding to the insulin receptor and initiate a signal transduction inside the cell. ${ }^{1,17,24}$ Many studies failed to find a relation between visfatin and IR in T2DM ${ }^{30,31}$ while many studies reported the presence of significant association with IR. ${ }^{32-}$

One of the important aims of the study is to investigate the correlation of visfatin with insulin sensitivity by the use of QUICKI for its simplicity and inexpensive useful index for clinical studies. ${ }^{35}$ Due to the documented limitation of HOMA IR as a tool for the estimation of IR because of the skewed distribution of FI values, the inverse logarithmic scale of HOMA IR; QUICKI is used to reflect the effect of insulin on hepatic glucose production. ${ }^{36}$

Interestingly, no correlation was reported between insulin sensitivity and plasma visfatin by many researchers who used hyper-insulinemic euglycemic clamp (insulin sensitivity standard evaluation method). ${ }^{15,32,37,38}$ Similarly, other study did not report correlation between visfatin and insulin sensitivity and lipid of intra-myocellular in skeletal muscle. ${ }^{5}$ Therefore, persuasive facts regarding the association between visfatin and IR in T2DM are still missing. Takebayashi et al., (2007) found no correlation between diabetes and levels of visfatin. ${ }^{39}$
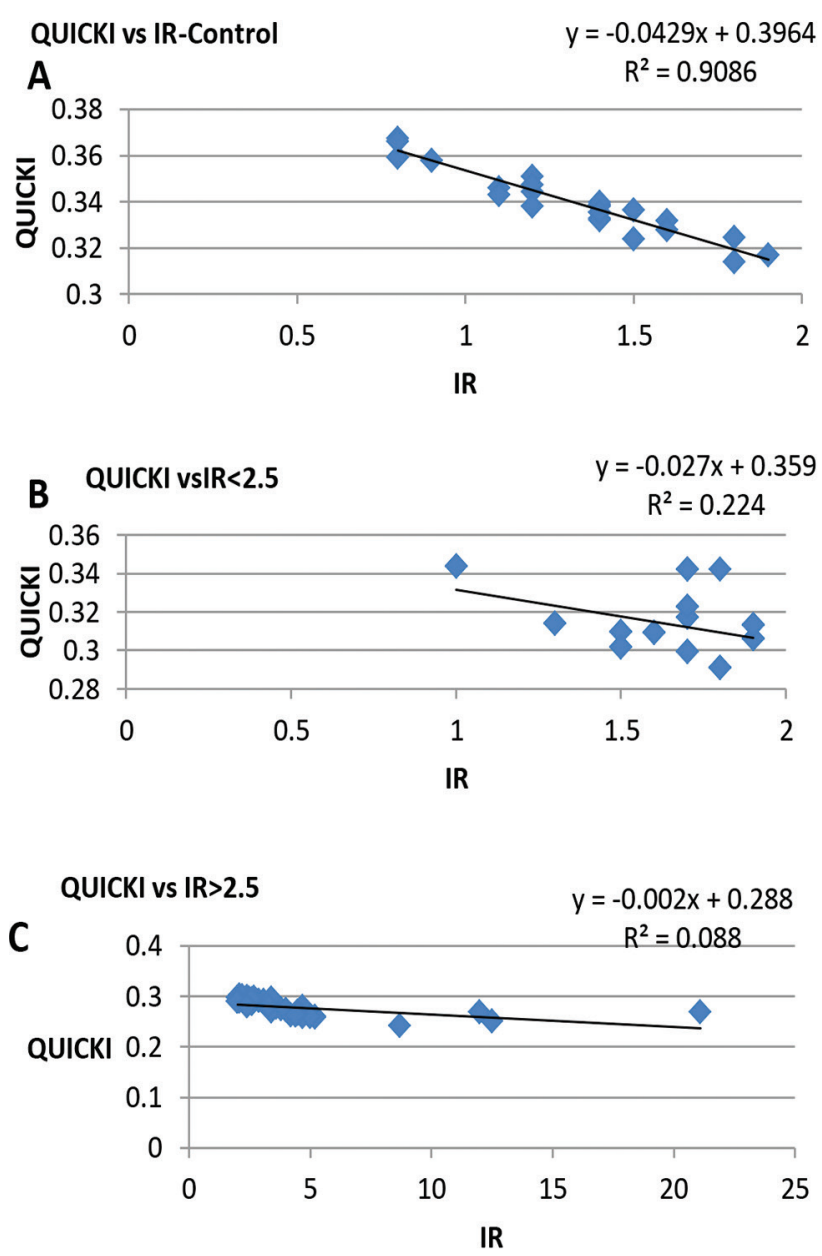

Fig 3. The Pearson correlation between QUICKI and IR in: A) control group, B) diabetic patients with IR $<2.5, C$ ) diabetic patients with IR $>2.5$.

\section{Conclusion}

The likelihood of elevation of visfatin in diabetic people is a mechanism to compensate insulin deficiency or its inefficiency, so that it can regulate blood glucose level at certain levels. It can be concluded that increase visfatin level may be inducible to regulate elevated glucose levels spatially when IR is 
more than 2.5. However, more studies with a larger number of patients are needed to confirm this conclusion.

\section{Acknowledgements}

Our deep gratitude is due to all patients, participants and staff members of laboratories in the National Centers for
Diabetes/AL-Mustansiriya University spatially Dr. Refif Sabih Al- Shawk for her assistant in collecting patient samples and their demographic information.

\section{Conflicts of Interest Disclosure}

There are no conflicts of interest.

\section{References}

1. Fukuhara A, Matsuda M, Nishizawa M, Segawa K, Tanaka M, Kishimoto K, et al. Visfatin: a protein secreted by visceral fat that mimics the effects of insulin. Science. 2005;307:426-430.

2. Garten A, Petzold S, Barnikol-Oettler A, Körner A, Thasler WE, Kratzsch J, et al. Nicotinamide phosphoribosyltransferase (NAMPT/PBEF/visfatin) is constitutively released from human hepatocytes. Biochem Biophys Res Commun. 2010;391:376-381.

3. Costford SR, Bajpeyi S, Pasarica M, Albarado DC, Thomas SC, Xie H, et al. Skeletal muscle NAMPT is induced by exercise in humans. Am J Physiol Endocrinol Metabol. 2010;298:E117-E126.

4. Curat CA, Wegner V, Sengenes C, Miranville A, Tonus C, Busse R, et al. Macrophages in human visceral adipose tissue: increased accumulation in obesity and a source of resistin and visfatin. Diabetologia. 2006;49:744.

5. Varma V, Yao-Borengasser A, Rasouli N, Bodles AM, Phanavanh B, Lee MJ, et al. Human visfatin expression: relationship to insulin sensitivity, intramyocellular lipids, and inflammation. J Clin Endocrinol Metabol. 2006;92:666-672.

6. Skop V, Kontrová K, Zídek V, Pravenec M, Kazdová L, Mikulík K, et al. Autocrine effects of visfatin on hepatocyte sensitivity to insulin action. Physiol Res. 2010;59:615.

7. Adeghate E. Visfatin: structure, function and relation to diabetes mellitus and other dysfunctions. Curr Med Chem. 2008;15:1851-1862.

8. Ognjanovic S, Bao S, Yamamoto SY, Garibay-Tupas J, Samal B, BryantGreenwood GD. Genomic organization of the gene coding for human preB-cell colony enhancing factor and expression in human fetal membranes. J Mol Endocrinol. 2001;26:107-117.

9. Moschen AR, Kaser A, Enrich B, Mosheimer B, Theurl M, Niederegger H, et al. Visfatin, an adipocytokine with proinflammatory and immunomodulating properties. J Immunol. 2007;178:1748-1758.

10. Wang T, Zhang X, Bheda P, Revollo JR, Imai SI, Wolberger C. Structure of Nampt/PBEF/visfatin, a mammalian NAD+ biosynthetic enzyme. Nat Struct Mol Biol. 2006;13:661

11. Khan JA, Xiao T, Liang T. Molecular basis for the inhibition of human NMPRTase, a novel target for anticancer agents. Nat Struct Mol Biol. 2006;13:582.

12. Rezk MY. Effect of visfatin on blood glucose and serum lipids in normal and streptozotocin induced diabetic Rats. Int J Anat Physiol. 2013;3:36-41.

13. Rezk MY. Visfatin and cardiovascular protection. J Drug Deliv Therap. 2014:4:154-166.

14. Ahmed HS, Tahir NT. Association between diabetes mellitus and knee osteoarthritis. Iraq Medical Journal. 2017;1:65-67.

15. Hammarstedt A, Pihlajamäki J, Rotter Sopasakis V, Gogg S, Jansson PA, Laakso M, et al. Visfatin is an adipokine, but it is not regulated by thiazolidinediones. J Clin Endocrinol Metabol. 2006;91:1181-1184.

16. Rabo SA, Mohammed NA, Eissa SS, Ali AA, Ismail SM, Gad RS. Serum visfatin in type 2 diabetes mellitus. Egyptian J Int Med. 2013;25:27.

17. Haider DG, Holzer G, Schaller G, Weghuber D, Widhalm K, Wagner O, et al. The adipokine visfatin is markedly elevated in obese children. J Pediatr Gastroenterol Nutr. 2006;43:548-549.

18. Dogru T, Sonmez A, Tasci I, Yilmaz MI, Erdem G, Erturk H, et al. Plasma visfatin levels in young male patients with uncomplicated and newly diagnosed hypertension. J Hum Hyperten. 2007:21:173-175.

19. Pagano C, Pilon C, Olivieri M, Mason P, Fabris R, Serra R, et al. Reduced plasma visfatin/pre-B cell colony-enhancing factor in obesity is not related to insulin resistance in humans. J Clin Endocrinol Metabol. 2006;91:3165-3170.

20. López-Bermejo A, Chico-Julià B, Fernàndez-Balsells M, Recasens M, Esteve E, Casamitjana R, et al. Serum visfatin increases with progressive $\beta$-cell deterioration. Diabetes. 2006;55:2871-2875.

21. Alberti KG, Zimmet PF. Definition, diagnosis and classification of diabetes mellitus and its complications. Part 1: diagnosis and classification of diabetes mellitus. Provisional report of a WHO consultation. Diabet Med. 1998;15:539-553.
22. Gutch M, Kumar S, Razi SM, Gupta KK, Gupta A. Assessment of insulin sensitivity/resistance. Indian J Endocrinol Metabol. 2015;19:160-164.

23. Katz A, Nambi SS, Mather K, Baron AD, Follmann DA, Sullivan G, et al. Quantitative insulin sensitivity check index: a simple, accurate method for assessing insulin sensitivity in humans. J Clin Endocrinol Metabol. 2000;85:2402-2410

24. Chen MP, Chung FM, Chang DM, Tsai JC, Huang HF, Shin SJ, et al. Elevated plasma level of visfatin/pre-B cell colony-enhancing factor in patients with type 2 diabetes mellitus. J Clin Endocrinol Metabol. 2006;91:295-299.

25. Jian WX, Luo TH, Gu YY, Zhang HL, Zheng S, Dai M, et al. The visfatin gene is associated with glucose and lipid metabolism in a Chinese population. Diabet Med. 2006:23:967-973.

26. Li L, Yang G, Li Q, Tang Y, Yang M, Yang H, et al. Changes and relations of circulating visfatin, apelin, and resistin levels in normal, impaired glucose tolerance, and type 2 diabetic subjects. Exp Clin Endocrinol Diabet. 2006;114:544-548.

27. Yaturu S, Davis J, Franklin L, Shi R, Venkatesh P, Jain SK. Visfatin levels are low in subjects with type 2 diabetes compared to age-matched controls. J Diabet Mellit. 2012;2:373-377.

28. Saddi-Rosa P, Oliveira CS, Giuffrida FM, Reis AF. Visfatin, glucose metabolism and vascular disease: a review of evidence. Diabetol Metabol Syndrome. 2010;2:21-26.

29. McGee KC, Harte AL, da Silva NF, Al-Daghri N, Creely SJ, Kusminski CM, et al. Visfatin is regulated by rosiglitazone in type 2 diabetes mellitus and influenced by NFKB and JNK in human abdominal subcutaneous adipocytes. PLoS one. 2011;6:e20287.

30. Esteghamati A, Alamdari A, Zandieh A, Elahi S, Khalilzadeh O, Nakhjavani $M$, et al. Serum visfatin is associated with type 2 diabetes mellitus independent of insulin resistance and obesity. Diabet Res Clin Pract. 2011:91:154-158.

31. Chang YH, Chang DM, Lin KC, Shin SJ, Lee YJ. Visfatin in overweight/ obesity, type 2 diabetes mellitus, insulin resistance, metabolic syndrome and cardiovascular diseases: a meta-analysis and systemic review. Diabet Metabol Res Rev. 2011;27:515-527.

32. Ognjanovic S, Jacobs DR, Steinberger J, Moran A, Sinaiko AR. Relation of chemokines to BMI and insulin resistance at ages 18-21. Int J Obes. 2013;37:420-423.

33. Wroblewski E, Swidnicka-Siergiejko A, Hady HR, Luba M, Konopko M, Kurek $\mathrm{K}$, et al. Variation in blood levels of hormones in obese patients following weight reduction induced by endoscopic and surgical bariatric therapies. Cytokine. 2016:77:56-62.

34. Agueda M, Lasa A, Simon E, Ares R, Larrarte E, Labayen I. Association of circulating visfatin concentrations with insulin resistance and low-grade inflammation after dietary energy restriction in Spanish obese non-diabetic women: role of body composition changes. Nutr Metabol Cardiovas Dis. 2012;22:208-214.

35. Patarrão RS, Lautt WW, Macedo MP. Assessment of methods and indexes of insulin sensitivity. Revista Portuguesa de Endocrinologia, Diabetes E Metabolismo. 2014;9:65-73.

36. Abbasi F, Reaven GM. Evaluation of the quantitative insulin sensitivity check index as an estimate of insulin sensitivity in humans. Metabol Clin Exp. 2002;51:235-237

37. Berndt J, Klöting N, Kralisch S, Kovacs P, Fasshauer M, Schön MR, et al. Plasma visfatin concentrations and fat depot-specific mRNA expression in humans. Diabetes. 2005;54:2911-2916.

38. Brema I, Hatunic M, Finucane F, Burns N, Nolan JJ, Haider D, et al. Plasma visfatin is reduced after aerobic exercise in early onset type 2 diabetes mellitus. Diabet Obes Metabol. 2008;10:600-602

39. Takebayashi K, Suetsugu M, Wakabayashi S, Aso Y, Inukai T. Association between plasma visfatin and vascular endothelial function in patients with type 2 diabetes mellitus. Metabolism. 2007;56:451-458.

This work is licensed under a Creative Commons Attribution-NonCommercial 3.0 Unported License which allows users to read, copy, distribute and make derivative works for non-commercial purposes from the material, as long as the author of the original work is cited properly. 